\title{
Rotational diffusion in dense suspensions
}

\author{
M.H.J. Hagen ${ }^{\mathrm{a}, *}$, D. Frenkel ${ }^{\mathrm{a}}$, C.P. Lowe ${ }^{\mathrm{b}}$ \\ ${ }^{a}$ FOM Institute for Atomic and Molecular Physics, Kruislaan 407, 1098 SJ Amsterdam, \\ The Netherlands \\ ${ }^{\mathrm{b}}$ Department of Computational Physics, Delft University of Technology, Lorentzweg 1, \\ 2628 CJ Delft, The Netherlands
}

Received 14 December 1998; received in revised form 23 June 1999

\begin{abstract}
We have computed the rotational diffusion coefficient for a suspension of hard spheres. We find excellent agreement with experimental results over a density range up to, and including, the colloidal crystal. However, we find that theories derived to second order in the volume fraction overestimate the rotational diffusion coefficient for volume fractions exceeding $25 \%$. To investigate the sensitivity of the rotational diffusion coefficient to the pair distribution function we also consider a perfect FCC crystal with negligible thermal motion. We show that, in line with theoretical predictions, the first term in the expansion of the rotational diffusion coefficient in powers of the volume fraction becomes quadratic. Relative to the random distribution, the rotational diffusion coefficient in this case is significantly larger. By studying the decay of angular velocity fluctuations, we examined the time dependence of the rotational diffusion coefficient. We find that for rotation the situation is similar to that reported for translation. The suspension behaves like an "effective fluid", i.e. the rotational dynamics of a particle in the suspension can be described by the isolated particle result, but with the suspension viscosity replacing the fluid viscosity. As with translation, this picture only holds for times long compared to the time it takes transverse momentum to diffuse over a distance of the order of a particle radius. (c) 1999 Elsevier Science B.V. All rights reserved.
\end{abstract}

PACS: $05.40 .+\mathrm{j} ; 51.20 .+\mathrm{d} ; 82.70 . \mathrm{Dd}$

\section{Introduction}

Whereas translational diffusion in concentrated colloidal suspensions of hard spheres has been extensively studied experimentally [1-4], values for the rotational diffusion coefficient have only been reported more recently $[5,6]$. Theoretically, as is the case for

\footnotetext{
* Corresponding author: present address: Unilever Research, Port Sunlight Laboratory, Quarry Road East, Bebington, Wirral CH63 3JW, UK.
} 
translation, the dilute limit is relatively straightforward and can be solved analytically. The rotational diffusion coefficient in this limit, $D_{0}^{R}$, is given by the Debye-Einstein relation [7]

$$
D_{0}^{R}=\frac{k_{B} T}{8 \pi \rho v R^{3}},
$$

where $\rho$ and $v$ are the density and kinematic viscosity of the solvent, respectively, $R$ the radius of the colloidal sphere, $T$ the temperature and $k$ Boltzmann's constant. For concentrated suspensions one can distinguish two well separated time regimes. Correlations in velocity fluctuations (angular and linear) decay on a time scale characterized by the diffusive transport of momentum through the solvent $\tau \sim R^{2} / v$. On the other hand, the time it takes a colloidal particle to move significantly relative to its neighbours, $\tau_{p}$, is of the order $R^{2} / D$, where $D$ is the short time translational diffusion coefficient. Putting in the numbers, for a typical suspension (particles of $1 \mu$ diameters dispersed in water) we find $\tau_{p} / \tau \sim 10^{6}$. Clearly, on the "short" time scale $\left(\tau_{p}>t>\tau\right)$ velocity correlations decay whilst the particles hardly move. In this case, only the many-body hydrodynamic interactions in an essentially "frozen" configurations of particles need be taken into account. This is the limit we consider here. For a given form of the pair distribution function, the short time transport coefficients can be expressed in the form of a "virial" expansion, in terms of the volume fraction $\phi$. The coefficients in this expansion can be derived from macroscopic hydrodynamics. For the rotational diffusion coefficient, $D^{R}$, in a suspension of colloidal hard spheres, Clercx and Schram [8] proposed that this calculation yields

$$
D^{R}=D_{0}^{R}\left(1-0.630 \phi-0.74 \phi^{2}\right) \text {. }
$$

A similar result was derived by Degiorgio et al. [5], although the coefficient for the second-order term differed somewhat (0.67 as opposed to 0.74$)$. In their article Degiorgio et al. [5] compared their experimental data with theoretical predictions for the rotational diffusion coefficient as a function of volume fraction. The experimental results compared well with the theory up to moderate volume fractions $(\phi \sim 0.3)$ and confirmed that, for rotation, it is essential to include the second-order term to describe the rotational diffusion coefficient at all but the lowest volume fractions. This contrasts with translational diffusion, where a first-order expansion adequately describes the diffusion coefficient up to quite high volume fractions $(\phi \sim 0.4)$. At higher volume fractions $(\phi>0.3)$, Degiorgio et al. found that the theory overestimated $D^{R}$. However, short time transport coefficients generally depend on the pair distribution function. In particular, the virial expansion for the rotational diffusion coefficient, Degiorgio et al. pointed out, is extremely sensitive to the form of the pair distribution function at small separations. In the experiments the colloidal particles are highly charged, but the electrostatic interactions are rendered short ranged by adding salt to the solvent. They can only, therefore, be regarded approximately as hard spheres. At high volume fractions it is possible that the short-ranged electrostatic interactions (and possibly even the polymer coats of the particles) influence the experimental values of the rotational diffusion coefficient. Thus, the question arises: does the disagreement found in Ref. [5] originate 
from the need to include a third-order term at high volume fractions or from an effect of the short-ranged potential between the colloidal particles used in the experiment? This question can most easily be addressed with the aid of a computer simulation.

Any effects of higher-order virial coefficients are more likely to be detected at high volume fractions. It would therefore be useful to calculate accurate values for the rotational diffusion coefficient of truly hard colloidal spheres right up to hard-sphere freezing $(\phi=0.49)$ and compare with the experimental values in the very dense regime. With this in mind, our first aim in this paper is to calculate rotational diffusion coefficients for truly hard spheres, up to hard-sphere freezing, and compare with the experimental values. This should enable us to shed some light on the question of whether the colloidal particles used in the experiment behaved as hard spheres at all volume fractions. The rotational diffusion coefficient for random distributions of hard spheres has been calculated numerically, up to volume fractions as high as $45 \%$, using both Stokesian dynamics [9] and the lattice Boltzmann method [10]. In both cases the calculation involved evaluating the average rotational mobility of the spheres, $\mu$, and then relating this to the rotational diffusion coefficient by way of the generalised Einstein equation [11].

$$
D^{R}=k_{B} T \mu .
$$

The experiments performed by Degiorgio et al. extended to the colloidal crystal regime (which has a face-centered cubic structure) for (almost) hard spheres. Suspensions of highly charged spheres, in the absence of added salt, crystallize at much lower volume fractions. Recently, Watzlawek and Nägele [12] studied theoretically a system of deionized charge stabilized particles at moderate volume fractions $(\phi<0.25)$. They found that

$$
D^{R}=D_{0}^{R}\left(1-1.15 \phi^{2}\right),
$$

a quadratic $\phi$ dependence. Note that in this case the $\phi$ dependence is quite different from that found for the random spheres (Eq. (2)). This clearly illustrates how sensitive the rotational diffusion coefficient is to the pair-distribution function. To gain an insight into the influence of the pair-distribution function on the rotational diffusion coefficient, we also consider what is effectively the opposite limit to randomly distributed hard spheres - namely, a perfect face centered cubic (FCC) crystal. By "perfect" we mean a crystal for which thermal motion can be neglected.

A second question that we can study with the aid of computer simulation is the transient rotational dynamics of a colloidal sphere. This tells us how the asymptotic rotational diffusion coefficient is reached and has, to our knowledge, not been studied experimentally. We probe the short-time rotational dynamics by studying the angular velocity autocorrelation function, $\psi(t)$, defined as

$$
\psi(t)=\left\langle\omega_{j}^{\alpha}(0) \omega_{j}^{\alpha}(t)\right\rangle,
$$

where $\alpha$ denotes one Cartesian component of the angular velocity $\omega$ and the averaging is carried out over all particles in the system. The rotational diffusion coefficient is 
related to the time integral of $\psi(t)$, so it is useful to define a "time-dependent" rotational diffusion coefficient, $D^{R}(t)$, by

$$
\frac{D^{R}(t)}{k_{B} T}=\frac{\int_{0}^{t} \psi(\tau) \mathrm{d} \tau}{I \psi(0)},
$$

where $I$ is the moment of inertia of the sphere. In the long time limit of the short time regime $\left(\tau \ll t<\tau_{p}\right)$ the time-dependent rotational diffusion coefficient approaches the short time rotational diffusion coefficient. In the interests of brevity we will subsequently refer to this regime as "long" times and denote it infinity, so that

$$
D^{R}=\lim _{t \rightarrow \infty} D^{R}(t) .
$$

Unlike rotation, the transient translational dynamics of colloidal suspensions have been studied experimentally [13], theoretically [14] and by computer simulation [15]. Whereas there is almost universal agreement regarding the value of the diffusion coefficient, its time dependence is still a matter for debate. One of our aims here is to see if the study of rotation in dense suspensions can shed some light on this matter.

To briefly recapitulate, the AVACF of a single-sphere decays asymptotically with an algebraic tail of the form [16]

$$
\psi(t \rightarrow \infty)=\psi(0) \frac{\pi I}{\rho}(4 \pi v t)^{-5 / 2} .
$$

There is a similar expression for the translational equivalent of the AVACF, the velocity autocorrelation function (VACF). The explicit form is given in Ref. [16], here it is sufficient to note that the algebraic decay is of the form $(v t)^{-3 / 2}$, i.e. one power slower. These results apply for a single sphere, i.e. in the dilute limit. It is natural to ask the question, what form does this decay take in a concentrated suspension? Intuitively one might expect that at sufficiently long times the concentrated suspension will behave like a fluid with the suspension viscosity (as opposed to the solvent viscosity). This "effective fluid" picture would suggest that, in a concentrated suspension, the decay of the AVACF (or the VACF, for that matter), will be described by the single particle result, but with the suspension viscosity replacing the solvent velocity. For translation, Milner and Liu [14] showed theoretically that, at least to order $\phi$ in the volume fraction, this was the case. Experiments [13,17] and computer simulations [15] of the VACF in concentrated suspensions confirmed this. The experiments, however, suggested that a concentrated suspension behaves like an 'effective fluid' on surprisingly short time scales. The hydrodynamic interactions between spheres, which modify the values of the transport coefficients in a concentrated suspension relative to those in the dilute limit, propagate by the diffusion of local momentum. This takes place on a time scale $t \sim \tau$. It would therefore be surprising if, as has been suggested [17], a suspension behaved like an effective fluid for times such that $t / \tau \ll 1$. In the case of the VACF, the analysis is complicated by the fact that the translational motion of the colloidal particle also couples to the longitudinal momentum (sound waves). Although this coupling is irrelevant at long times, it partially masks the effect of the transverse momentum at short times. For the AVACF, sound waves play no role. Hence, by studying the short-time 
behaviour of the AVACF in a suspension we can determine on what time scale the diffusion of transverse momentum has progressed to the point where the suspension starts to behave like an effective fluid.

\section{Description of the model}

In this section we outline the lattice-Boltzmann simulation method and how we applied it to compute the rotational diffusion coefficient of a suspension of colloidal hard spheres. The method, historically at least, is a pre-averaged version of a latticegas cellular automaton (LGCA) model of a fluid. In lattice-gas cellular automaton the state of the fluid at any (discrete) time is specified by the number of particles at every lattice site and their velocity. Particles can only move in a limited number of directions (towards neighbouring lattice points) and there can be at most one particle moving on a given 'link'. The time evolution of the LGCA consists of two steps - 1. Propagation: every particle moves in one time step, along its link to the next lattice site. 2. Collision: at every lattice site particles can change their velocities by collision, subject to the condition that these collisions conserve number of particles and momentum (and retain the full symmetry of the lattice). In the lattice-Boltzmann method (see e.g. [18]) the state of the fluid system is no longer characterised by the number of particles that move in direction $\mathbf{c}_{i}$ on lattice site $\mathbf{r}$, but by the probability to find such a particle. The single-particle distribution function $n_{i}(\mathbf{r}, t)$, describes the average number of particles at a particular node of the lattice $\mathbf{r}$, at a time $t$, with the discrete velocity $\mathbf{c}_{i}$. The hydrodynamic fields, mass density $\rho$, momentum density $\mathbf{j}$, and the momentum flux density $\boldsymbol{\Pi}$ are simply moments of this velocity distribution:

$$
\rho=\sum_{i} n_{i}, \quad \mathbf{j}=\sum_{i} n_{i} \mathbf{c}_{i}, \quad \boldsymbol{\Pi}=\sum_{i} n_{i} \mathbf{c}_{i} \mathbf{c}_{i} .
$$

The lattice model used in this work is the four-dimensional face-centered hyper cubic (FCHC) lattice. A three-dimensional model can then be obtained by projection. This FCHC model is used because three-dimensional cubic lattices do not have a high enough symmetry to ensure that the hydrodynamic transport coefficients are isotropic.

The time evolution of the distribution functions $n_{i}$ is described by the discretized analogue of the Boltzmann equation [19]:

$$
n_{i}\left(\mathbf{r}+\mathbf{c}_{i}, t+1\right)=n_{i}(\mathbf{r}, t)+\Delta_{i}(\mathbf{r}, t),
$$

where $\Delta_{i}$ is the change in $n_{i}$ due to instantaneous molecular collisions at the lattice nodes. The post-collision distribution $n_{i}+\Delta_{i}$ is propagated in the direction of the velocity vector $\mathbf{c}_{i}$. A complete description of the collision process is given in [18]. The main effect of the collision operator $\Delta_{i}(\mathbf{r}, t)$ is to (partially) relax the shear stress at every lattice site. The rate of stress relaxation or equivalently, the kinematic viscosity $v$ can be chosen freely.

The motion of the colloidal particle is determined by the force and torque exerted on it by the fluid. These are in turn a result of the stick boundary conditions applied at the 
solid/fluid interface. For a stationary boundary a simple bounce-back rule performed on boundary links enforces the stick boundary condition. Boundary links are links connecting lattice sites inside and outside the solid object. For a moving boundary the bounce back rule is still applied but some of the particles moving in the same direction as the solid object are allowed to "leak" through, thus matching the fluid velocity to the object velocity at the boundary [18]. The equations of motion of the colloidal particles are then integrated according to a rule [20] whereby the force and torque which act on each object give the same new velocities for both particle and fluid. This approach is unconditionally stable and allows the mass and moment of inertia to be chosen freely.

Rather than evaluating the rotational mobility of each particle in the system and using the generalised Einstein equation (3) (the approach used by Behrend [10] to obtain the rotational diffusion coefficient), we make use of Onsager's regression hypothesis and study the decay of an impulsive angular velocity perturbation in the otherwise dissipative system (there are no spontaneous fluctuations). The impulsive perturbation is generated by assigning an initial angular velocity $\omega_{j}(0)$, taken from a MaxwellBoltzmann distribution, to each particle. The subsequent time evolution of the angular velocity $\omega_{j}(t)$ is then computed and hence the AVACF. From the AVACF the rotational diffusion coefficient is evaluated using Eq. (7). These two methods may appear quite different but are shown in the appendix to be equivalent. The advantages of the methodology we employ are twofold. First, it enables us to calculate the AVACF as well as the value of the rotational diffusion coefficient, thus we glean extra information. Second, simply viewed as a means of calculating the rotational diffusion coefficient, it is more efficient. Rather than evaluating the mobility of an individual particle, one essentially calculates the average mobility for all $N$ particles in the system in a simulation of roughly equivalent duration. Further averaging was performed by repeating this procedure for a number of independent configurations of spheres.

\section{Results}

Starting from a lattice-Boltzmann fluid initialised at rest, we used the method outlined above to follow the time evolution of the angular velocity correlation function of the suspended particles. In all the simulations the viscosity of the fluid was equal to $v_{0}=\frac{1}{6}$ and the density $\rho$ was 24 (all quantities are given in lattice units, where the mass of the lattice-gas particles, the lattice spacing and the time step are all unity). Both the mass and moment of inertia of the spheres were assigned values appropriate for a sphere with a uniform density equal to that of the fluid.

In order to obtain the normalised rotational diffusion coefficient $\left(D^{R} / D_{0}^{R}\right)$ one also needs to determine $D_{0}^{R}$. In principle, this can be done analytically, using the DebyeEinstein relation, but in practice $D_{0}^{R}$ in the simulation has a slightly different value (because of the approximation involved in mapping a sphere onto a cubic lattice). In order to compensate for this error we calculated the infinite dilution value of the rotational diffusion coefficient by numerically integrating the AVACF of a single sphere. 


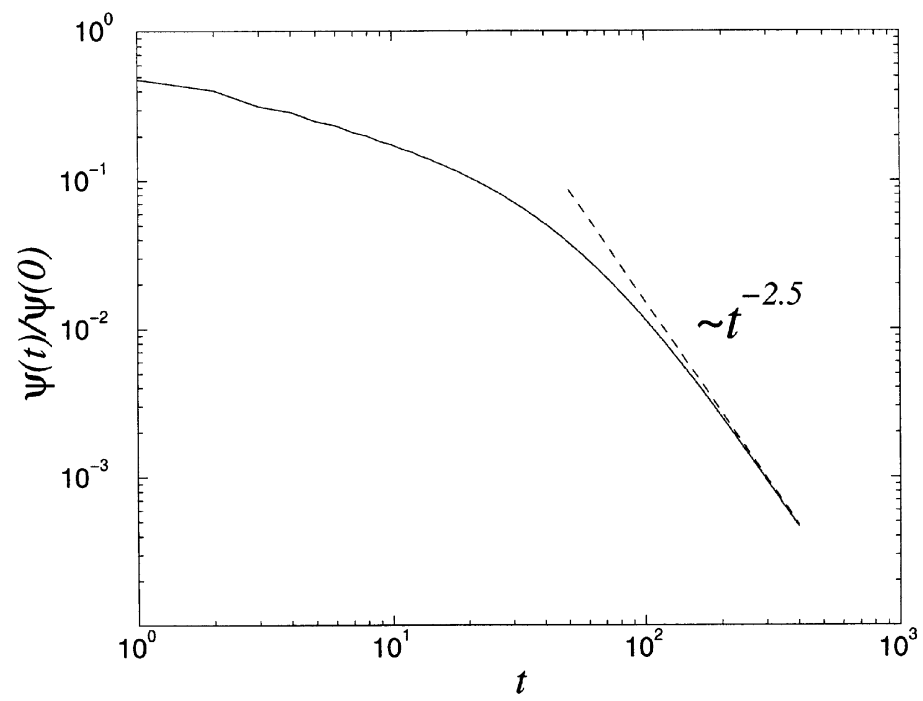

Fig. 1. Normalised angular velocity correlation function for a single suspended sphere of radius $R=4.5$ (lattice units). Time is expressed in units of the discrete time step used in the lattice model. The solid line denotes the simulation result, the dashed line denotes the theoretical long-time behaviour.

The single particle rotational diffusion coefficient thus determined can be used to fix a "hydrodynamic" or "effective" radius for the colloidal particle [18]. For a sphere of nominal radius $R=4.5$ (lattice units) the hydrodynamic radius we calculated was only $1.5 \%$ larger than the nominal radius. When we move on to consider concentrated suspension this error is small compared to other sources of error so, for spheres with nominal radius 4.5 or greater, the difference between the nominal and hydrodynamic radius will be neglected and the radius taken to be the nominal radius. The normalised angular velocity correlation function and the predicted long time behaviour (Eq. (8)) are shown in Fig. 1. We see that the predicted power $(-2.5)$ and amplitude of the long time behaviour (from Eq. (8)) are both accurately reproduced by the simulation. This simulation was performed using a sphere of nominal radius 4.5 , the simulation box being replicated periodically in space. By comparing results for simulations using different system sizes, but otherwise identical, we concluded that the boundary conditions have very little effect on the AVACF (less than a percent) for times shorter than the time it takes transverse momentum to diffuse distances of the order of the linear dimension of the simulation box, that is $t_{b o x} \sim L^{2} / v$. This is in contrast to translation, where sound propagation is the dominant effect and thus $t_{b o x} \sim L / c_{s}$, where $c_{s}$ is the speed of sound (equal to $1 / \sqrt{ } 2$ in our model). Since, for typical parameters used in the simulations, the former is much longer than the latter, it is easier to exclude the effects of the periodic boundary conditions on the AVACF than the VACF. This makes it practical to study rotation, free from boundary artifacts, for longer times than translation [15]. We have excluded finite size effects from all the results we describe here by limiting our attentions to times such that $t<t_{b o x}$. 
For the concentrated suspensions we generated non-overlapping hard sphere configurations at the desired volume fraction using standard Monte Carlo techniques [21]. The angular velocity autocorrelation function was calculated for each configuration and the results then averaged over a number of independent hard-sphere configurations. The simulations were performed for solid volume fractions $\phi=0.05,0.10, \ldots, 0.45,0.49,0.55$. The highest two densities are those of the coexisting phases of the hard sphere model at melting [22]. The last $(\phi=0.55)$ case is the FCC-solid. The configurations for the FCC solid were also generated using Monte Carlo techniques. The spheres were not placed on a perfect FCC lattice but on a thermally equilibrated FCC lattice. That is, we allow for the effects of lattice vibrations. There are two sources of error in our simulations. First, finite size effects, which we have excluded by adopting the procedure outlined above. The second (and more problematic) is inability of the simulations to resolve the hydrodynamic interactions accurately between spheres in close proximity. The lattice Boltzmann approach will inevitably give a poor approximation to the true hydrodynamic forces when surfaces are separated by distance of the order of the lattice spacing or less. The only way around this problem is to repeat the calculation with a higher spatial resolution (i.e. increase the nominal radius, measured in units of the lattice spacing, of the spheres). Thus, the procedure we adopted was, at all densities, to repeat the simulations with progressively larger spheres until the results became independent of the sphere radius (or, more strictly, that the variation we observed on changing the sphere size was of the same order as the error associated with averaging over a number of configurations). For the simulations at low densities $(\phi<0.3)$ a relatively small sphere suffices. A large number of small spheres were then simulated, a large system being required to allow us to calculate the AVACF (free from boundary effects) for long enough times to gain a good estimate for the rotational diffusion coefficient. At higher volume fractions finite size effects become less important (because the rapidly increasing viscosity of the suspension causes a more rapid decay of the AVACF) but a large representation of the spheres is required. As a result the emphasis at high volume fraction shifts from a large number of small spheres to a smaller number of large spheres. At all solid volume fractions we calculated the normalised angular velocity auto correlation function $(\psi(t) / \psi(0))$, and, by numerical integration, the normalised time-dependent rotational diffusion coefficient $D^{R}(t) / D_{0}^{R}$. The limiting value of this function was estimated by plotting $D^{R}(t) / D 0^{R}$ as a function of $1 / t^{3 / 2}$, the intercept at $1 / t=0$ yielding the long time asymptote and hence the rotational diffusion coefficient. The extrapolation was performed by fitting a power series up to second order. In Table 1 we show the simulation results and the relevant parameters used in the simulations at various volume fractions. Taking all sources of error into account, these parameters were necessary to obtain values of $D^{R} / D_{0}^{R}$ to an accuracy of $4 \%$ or better. In Fig. 2 we have plotted the values we obtained for $D^{R} / D_{0}^{R}$ as a function of $\phi$, along with experimentally measured values [5,6]. Clearly, the agreement is very good over the entire density range. The simulations also show the apparent discontinuity upon going from the glass to the crystal, i.e. the value for the colloidal crystal lies above the value one would expect based on the trend for the glass. In Fig. 3 we 
Table 1

Simulation results and parameters. $\phi$ is the solid volume fraction, $R$ is the radius of the spheres used (in units of the lattice spacing), $N$ is the number of spheres and $D^{R} / D_{0}^{R}$ is the normalised rotational diffusion coefficient

\begin{tabular}{lrrl}
\hline$\phi$ & $R$ & \multicolumn{1}{l}{$N$} & $D^{R} / D_{0}^{R}$ \\
\hline 0.05 & 2.5 & 1320 & 0.97 \\
0.10 & 2.5 & 2640 & 0.94 \\
0.15 & 4.5 & 679 & 0.90 \\
0.20 & 4.5 & 905 & 0.85 \\
0.25 & 4.5 & 1132 & 0.80 \\
0.30 & 6.5 & 216 & 0.73 \\
0.35 & 5.5 & 108 & 0.67 \\
0.40 & 5.5 & 108 & 0.60 \\
0.45 & 9.5 & 108 & 0.53 \\
0.49 & 12.5 & 108 & 0.50 \\
0.55 & 13.5 & 108 & 0.47 \\
\hline
\end{tabular}

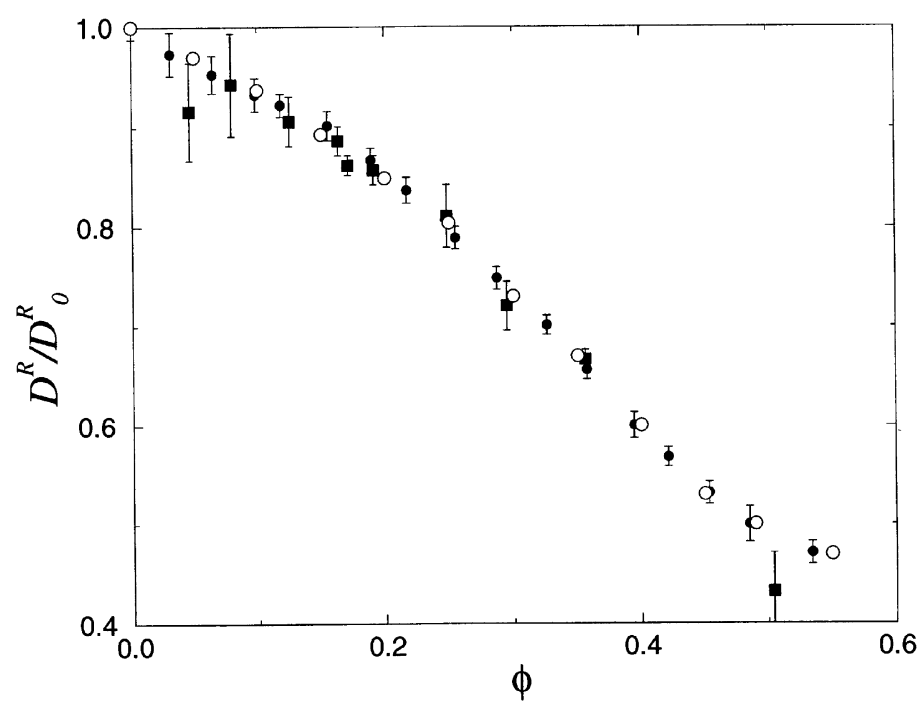

Fig. 2. Rotational diffusion coefficient as a function of volume fraction. The open circles are the simulation results. The filled circles with error bars are the experimental values from Ref. [5] and the filled squares are the results from Ref. [6].

show the comparison with the relevant virial expressions from Refs. $[5,8]$. There is (to within the statistical uncertainty) agreement for volume fractions $\phi<0.30$. By fitting the simulation data for $\phi \leqslant 0.2$ to a quadratic function we find

$$
D^{R}=D_{0}^{R}\left(1-(0.63 \pm 0.02) \phi-(0.68 \pm 0.08) \phi^{2}\right) .
$$

Thus, the value of the first virial coefficient is consistent with theory $[5,8]$. The value for the second coefficient is closer to the value of 0.67 derived by Degiorgio et al. than the value 0.74 derived by Clercx and Schram. However, both values lie within 


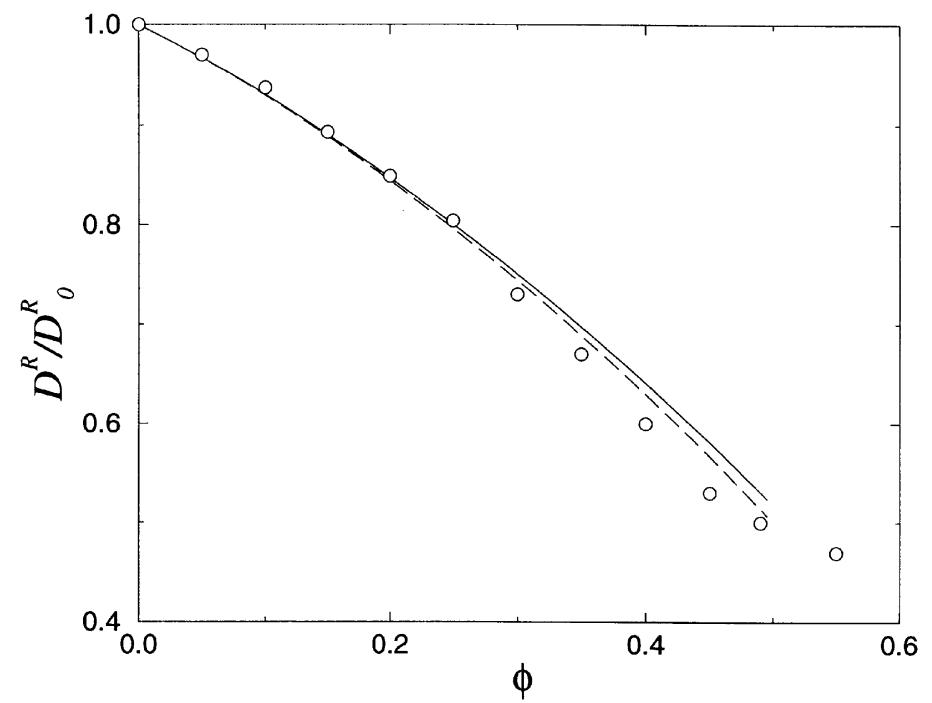

Fig. 3. Rotational diffusion coefficient as a function of volume fraction. The open circles are the simulation results. The solid line is the result derived in Ref. [5] and the dashed line the result derived in Ref. [8].

the range of uncertainty so we cannot unambiguously differentiate between the two. At higher densities both theories overestimate the true rotational diffusion coefficient. This must reflect the neglect of the $\phi^{3}$ term in the expansion.

We now proceed with the investigation of the influence of the pair distribution function on the rotational diffusion coefficient. To do so we located the colloidal spheres on a regular (non-thermally equilibrated) FCC lattice. The simulations were then repeated over the same range of densities used for the thermally equilibrated suspensions. The values for the rotational diffusion coefficient we calculated from the simulations are plotted in Fig. 4, along with the theoretical prediction from Eq. (4). Fitting our numerical data for $D^{R} / D_{0}^{R}$ to a polynomial in $\phi(\phi \leqslant 0.30$, the same range used in Ref. [12]), we find

$$
D^{R}=D_{0}^{R}\left(1+(0.03 \pm 0.03) \phi-(1.22 \pm 0.15) \phi^{2}\right) .
$$

The pre-factor of the linear term is small (and positive, which is unphysical), and our data are thus best described by a polynomial that contains no term linear in $\phi$. The quadratic term agrees (within statistical uncertainty) with the value of 1.15 predicted theoretically by Watzlawek and Nägele [12].

We now turn to the question of the time dependence of the hydrodynamic interactions. To do so we focus on one particular intermediate volume fraction, $\phi=0.20$. For a suspension of $N=1438$ nominally neutrally buoyant spheres with radius $R=4.5$ suspended in a fluid with viscosity $v_{0}=\frac{1}{6}$ we calculated the angular velocity correlation function, $\psi(t)$. If the suspension behaves like an effective fluid then we expect, based on Eq. (8), that the long time decay of AVACF will take the form

$$
\psi(t \rightarrow \infty) / \psi(0)=\frac{\pi I}{\rho^{*}}\left(4 \pi v_{\phi} t\right)^{-5 / 2},
$$




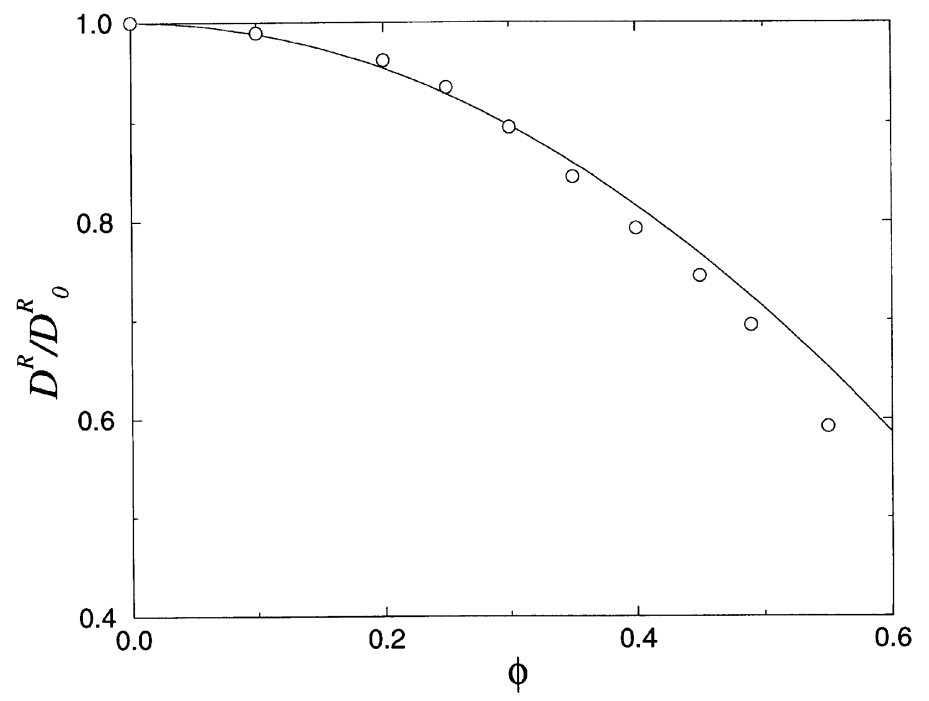

Fig. 4. Rotational diffusion coefficient as a function of volume fraction for spheres located on a regular FCC lattice. The solid line is Eq. (4).

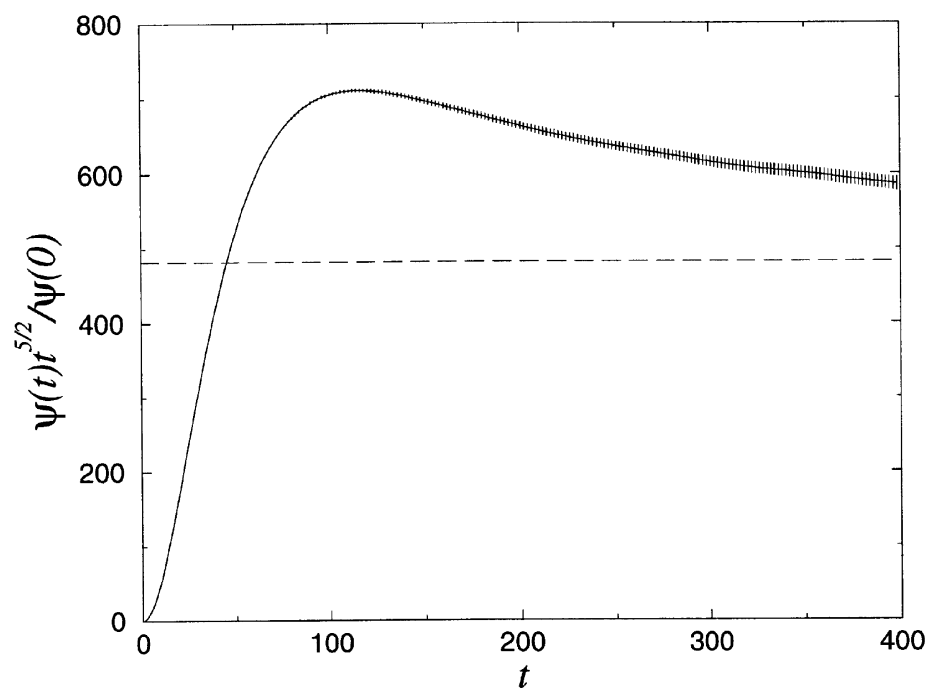

Fig. 5. Plot of $\psi(t) t^{5 / 2} / \psi(0)$ for a suspension with volume fraction $\phi=0.20$. The dashed line denotes $\lim _{t \rightarrow \infty} \psi(t) t^{5 / 2} / \psi(0)$. Time is expressed in units of the discrete time step used in the lattice model.

where $v_{\phi}$ is the suspension viscosity and $\rho^{*}=\rho(1+\phi)$. The latter represents a correction for the rotational inertia of the fluid inside the spheres (which is a small computational artifact). In Fig. 5 we have plotted the AVACF for this system, multiplied by $t^{5 / 2}$. If the long time decay takes the form we expect (Eq. (13)) this plot should approach a constant at long times. From the plot it does appear to be approaching a constant but the constant value has not been reached. To allow for this, and attempt to extract 


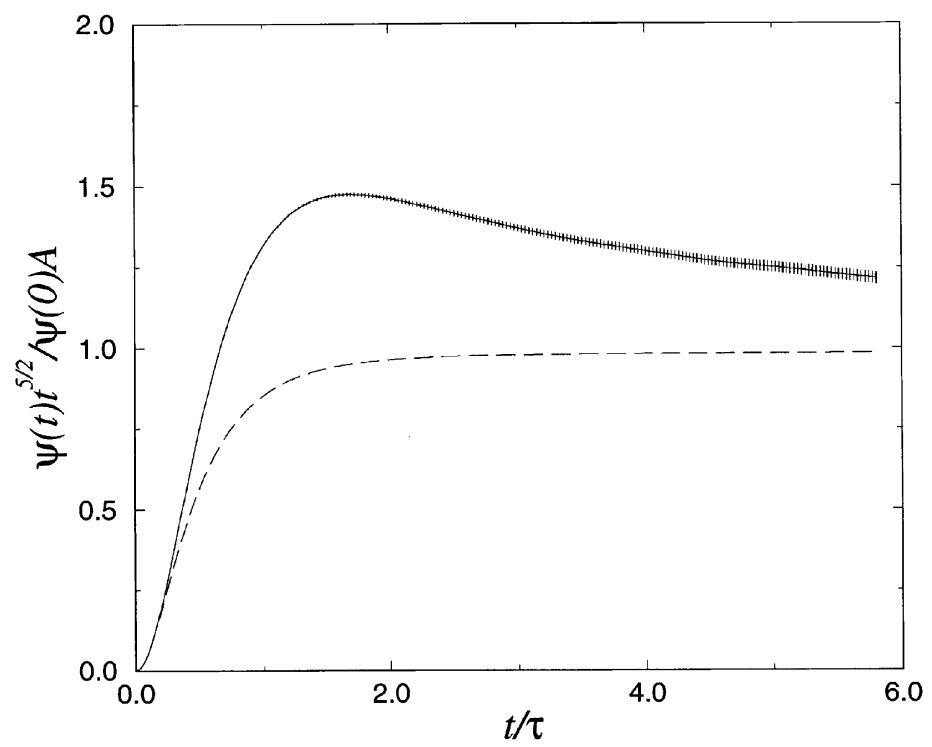

Fig. 6. Plot of $\psi(t) t^{5 / 2} / \psi(0) A$ for a suspension with volume fraction $\phi=0.20$ (solid line), and for a single particle (dashed line). Time is expressed in units of the hydrodynamic time $\tau=R^{2} / v_{\phi}$, where $v_{\phi}$ is the solvent viscosity for the single particle and the suspension viscosity for $\phi=0.20$. $A$ is the asymptotic value of $\psi(t) t^{5 / 2} / \psi(0)$.

the correct value for the constant, we adopted a procedure of extrapolating to longer times. We expect that, taking into account higher-order terms, the long-time decay can be expanded as a polynomial of the form $\psi(t) t^{5 / 2} / \psi(0) \sim A+B / t+C / t^{2}$. By fitting a polynomial of this form to the data shown in Fig. 5 we obtain a plateau value of $\psi(t \rightarrow$ $\infty) t^{5 / 2} / \psi(0)=A=482 \pm 10$, where the statistical error includes both errors associated with ensemble averaging and uncertainty in the extrapolation. We can now substitute this value into Eq. (13) and extract an apparent viscosity based on making the effective fluid assumption. This procedure yields a value $v_{\phi} / v_{0}=1.77 \pm 0.04$. This compares with the accurate numerical value, calculated by Ladd [23], of $v_{\phi} / v_{0}=1.81 \pm 0.02$. We can therefore conclude that the effective fluid argument describes our data very well - at least, after sufficiently long times. To be more precise as to what we mean by "sufficiently" long, we need to compare the AVACF for a colloidal particle in a suspension with the single-particle function scaled to the appropriate viscosity. To this end, in Fig. 6 we have plotted the normalised AVACF calculated for the suspension, multiplied by $t^{5 / 2}$ and divided by the long time asymptote, A, as a function of a reduced time $t / \tau$, where $\tau=R^{2} / v_{\phi}$. On the same graph we show the same function for a single particle (in this case $\tau=R^{2} / v_{0}$, i.e. the time is measured in units defined by the solvent rather than the suspension viscosity). It is important to note that following this procedure everything is dimensionless and the single particle curve is unique. Therefore, if the AVACF in a suspension can be described by the AVACF of a single particle in a solvent with the suspension viscosity, the two curves should coincide. Clearly, for the range of times we studied, they do not. This is despite the fact that, by 
construction, the two functions approach the same asymptote. We can thus conclude that only for long times where $t / \tau \gg 1$ does the effective fluid argument hold. More specifically, it holds for times where the decay of the AVACF in the suspension can be described by its asymptotic decay. As Fig. 6 shows, this time is markedly longer for the suspension than for the single particle. This is precisely the same conclusion arrived at for translation [15], although, comparing Fig. 6 with the equivalent plot for translation (Ref. [15]), the conclusion is much more clear-cut for the rotational case.

\section{Conclusions}

We have calculated short time rotational diffusion coefficient for hard spheres over the full range of volume fractions. We find agreement with experimental results at all volume fractions. It was suggested [5] that a theory valid to order $\phi^{2}$ might be valid up to hard sphere freezing, but with a modified pair distribution function. At high volume fractions, the suggestion was, the colloidal particles did not behave as truly hard spheres. Our simulations showed this was not the case and that the experimental values for the rotational diffusion coefficient were consistent with true hard sphere behaviour in the dense fluid, the glass and the colloidal crystal regimes. We can thus conclude that the difference between theory and experiment at high volume fractions, reported in Ref. [5], stems solely from the need to include higher-order terms in the viral expansion. Our simulations did, however, confirm that the rotational diffusion coefficient is very sensitive to the form of the pair distribution function. We only found agreement with experiment for the colloidal crystal if we allowed for the effect of thermal fluctuations of the particle positions about their equilibrium values. This point was emphasised when we studied the perfect FCC structure and found that, when compared to experiment, it gave a poor estimate for the rotational diffusion coefficient in a colloidal glass. Studying the perfect FCC crystal as a function of volume fraction, we were able to show that, for this system, the order $\phi$ term is zero, in line with theoretical predictions. Further, the order $\phi^{2}$ term is well described by the theory of Ref. [12].

When we examined the transient rotational dynamics, by calculating the angular velocity autocorrelation function, we found that the decay of the AVACF in a suspension had an algebraic decay of the same form found in the dilute limit. Further, we found this algebraic tail could be quantitatively described by the single particle result if we simply replaced the solvent viscosity with the suspension viscosity. Having established the asymptotic functional form of the decay of the AVACF, it should be possible to test this observation by making a direct comparison with experimental results obtained for times longer than those accessible in the simulations. Comparing the full time dependence of the scaled single-particle function with the AVACF in a concentrated suspension, we found that the the scaled function only described the decay adequately in the long-time limit. At shorter times the AVACF had a different functional form to the single particle case. This reflects the fact that on these very short time-scales, 
during which the hydrodynamic interactions are propagating, the suspension does not behave like an "effective" Newtonian fluid.

\section{Acknowledgements}

The work of the FOM Institute is part of the scientific program of FOM and is supported by the Nederlandse Organisatie voor Wetenschappelijk Onderzoek (NWO). Computer time on the CRAY-C98/4256 at SARA was made available by the Stichting Nationale Computer Faciliteiten (Foundation for National Computing Facilities). We gratefully acknowledge P. Bladon and P. Bolhuis for generation of hard sphere configurations. We thank I. Pagonabarraga for his useful comments and critical reading of the manuscript and R. Piazza for useful discussions of the experimental results. The research of Dr. Lowe has been made possible by a fellowship of the Royal Netherlands Academy of Arts and Sciences.

\section{Appendix A}

In this appendix we show that our method for calculating the rotational diffusion in a purely dissipative system (where there are no spontaneous fluctuations), is equivalent both to calculating it in a "real" dissipative system or proceeding via the generalised Einstein equation.

\section{A.1. The fluctuating system}

In a real colloidal suspension the dynamics of the colloidal particles and the solvent particles evolve according to Newton's Laws of motion. However, projecting out the fast degrees of freedom associated with the solvent, the motion of the colloidal particles can be shown to be described by a generalised Langevin equation [24-26]. For one component of the angular velocity this takes the form

$$
I \frac{\mathrm{d} \omega_{i}}{\mathrm{~d} t}=-\int_{0}^{t} \sum_{j} \xi_{i j}(t-s) \omega_{j}(s) \mathrm{d} s+T_{i}(t),
$$

where $I$ is the moment of inertia, $\xi_{i j}$ is a time-dependent friction matrix, and $T_{i}$ is the random torque, which is by definition orthogonal to the angular velocity. Taking the Laplace transform

$$
I\left(z \tilde{\omega}_{i}(z)-\omega_{i}(0)\right)-\tilde{T}_{i}(z)=-\sum_{j} \tilde{\xi}_{i j}(z) \tilde{\omega}_{j}(z),
$$

where $z$ is the Laplace transform variable, and $\omega_{i}(0)$ is the angular velocity at time zero. Transformed quantities are indicated with by a tilde. If we set $z=0$ we find

$$
I \omega_{i}(0)+\tilde{T}_{i}(0)=\sum_{j} \tilde{\xi}_{i j}(0) \tilde{\omega}_{j}(0),
$$


or as a matrix equation

$$
I \omega(0)+\tilde{T}(0)=\tilde{\xi}(0) \tilde{\omega}(0) .
$$

Inverting the equation and taking the product with $\omega(0)$ we find

$$
\omega(0) \tilde{\omega}(0)=\omega(0) \tilde{\xi}(0)^{-1}(I \omega(0)+\tilde{T}(0)) .
$$

Or in terms of individual components

$$
\frac{1}{N} \sum_{i} \omega_{i}(0) \tilde{\omega}_{i}(0)=\frac{1}{N} \sum_{i j}\left(\tilde{\xi}(0)^{-1}\right)_{i j}\left(I \omega_{i}(0) \omega_{j}(0)+\omega_{i}(0) \tilde{T}_{j}(0)\right) .
$$

If we now take the velocity average $\langle\cdots\rangle$, and note that for short-time diffusion the friction matrix $\xi$ only depends on the instantaneous positions of the particles, we find

$$
\frac{1}{N} \sum_{i}\left\langle\omega_{i}(0) \tilde{\omega}_{i}(0)\right\rangle=\frac{1}{N} \sum_{i j}\left(\tilde{\xi}(0)^{-1}\right)_{i j}\left(I\left\langle\omega_{i}(0) \omega_{j}(0)\right\rangle+\left\langle\omega_{i}(0) \tilde{T}_{j}(0)\right\rangle\right) .
$$

In an equilibrium system we know from thermodynamics that $I\left\langle\omega_{i}(0) \omega_{j}(0)\right\rangle=k_{B} T \delta_{i j}$ and by definition we have $\left\langle\omega_{i}(0) \tilde{T}_{j}(0)\right\rangle=0$, hence

$$
D^{R}=\frac{1}{N} \sum_{i}\left\langle\omega_{i}(0) \tilde{\omega}_{i}(0)\right\rangle=\frac{k_{B} T}{N} \sum_{i}\left(\tilde{\xi}(0)^{-1}\right)_{i i}=k_{B} T \mu,
$$

which is the generalised Einstein equation $\left((1 / N) \sum_{i}\left(\tilde{\xi}(0)^{-1}\right)_{i i}\right.$ is by definition the average mobility). This shows that the short-time rotational diffusion coefficient is the trace of the corresponding part of the mobility matrix. Now we describe how we effectively compute the average mobility matrix.

\section{A.2. The dissipative system}

The dynamics of the purely dissipative system we study in the simulation are described by an identical Langevin equation, but this time without the random torque, i.e.

$$
I \frac{\mathrm{d} \omega_{i}}{\mathrm{~d} t}=-\int_{0}^{t} \sum_{j} \xi_{i j}(t-s) \omega_{j}(s) \mathrm{d} s
$$

Again, taking the Laplace transform

$$
\omega(0) \tilde{\omega}(0)=I \omega(0) \tilde{\xi}(0)^{-1} \omega(0),
$$

or in terms of individual components

$$
\frac{1}{N} \sum_{i} \omega_{i}(0) \tilde{\omega}_{i}(0)=\frac{1}{N} \sum_{i j}\left(\tilde{\xi}(0)^{-1}\right)_{i j} I \omega_{i}(0) \omega_{j}(0) .
$$


If we now specify the statistical properties of the initial angular velocities velocities such that $I\left\langle\omega_{i}(0) \omega_{j}(0)\right\rangle=k_{B} T \delta_{i j}$, denoted $[\cdots]$, then we find that

$$
\frac{k_{B} T}{N} \sum_{i}\left(\tilde{\xi}(0)^{-1}\right)_{i i}=\frac{1}{N} \sum_{i}\left[\omega_{i}(0) \tilde{\omega}_{i}(0)\right] .
$$

The right-hand side of this equation is the quantity that we calculate in the simulation. Comparing with Eq. (A.8), which we derived for the "real" system, then this is clearly equal to the rotational diffusion coefficient. So to summarise, by this approach we can calculate the rotational diffusion coefficient in the dissipative system, without needing to average over thermal fluctuations, and without having to explicitly evaluate the mobility on a particle-by-particle basis.

\section{References}

[1] Y.W. Kim, J.E. Matta, Phys. Rev. Lett. 31 (1973) 208.

[2] P.D. Fedele, Y.W. Kim, Phys. Rev. Lett. 44 (1980) 691.

[3] G.L. Paul, P.N. Pusey, J. Phys. A 14 (1981) 3301.

[4] Chr. Morkel, Chr. Gronemeyer, W. Gläser, J. Bosse, Phys. Rev. Lett. 58 (1987) 1873.

[5] V. Degiorgio, R. Piazza, R.B. Jones, Phys. Rev. E. 52 (1995) 2707.

[6] J. Kanetakis, A. Tölle, H. Sillescu, Phys. Rev. E 55 (1997) 3006.

[7] P. Debye, Polar Molecules, Dover, New York, 1929.

[8] H.J.H. Clercx, P.P.J.M. Schram, J. Chem. Phys. 96 (1992) 3137.

[9] R.J. Phillips, J.F. Brady, G. Bossis, Phys. Fluids 31 (1988) 3462.

[10] O. Behrend, Phys. Rev. E. 52 (1995) 1164.

[11] P. Mazur, Faraday Discuss. Chem Soc. 83 (1987) 33.

[12] M. Watzlawek, G. Nägele, Physica A 235 (1997) 56.

[13] J.X. Zhu, D.J. Durian, J. Müller, D.A. Weitz, D.J. Pine, Phys. Rev. Lett. 68 (1992) 2559.

[14] S.T. Milner, A.J. Liu, Phys. Rev. E 48 (1993) 449.

[15] C.P. Lowe, D. Frenkel, Phys. Rev. E. 54 (1992) 2704.

[16] E.H. Hauge, A. Martin-Löf, J. Stat. Phys. 7 (1973) 259.

[17] M.H. Kao, A.G. Yodh, D.J. Pine, Phys. Rev. Lett. 70 (1993) 242.

[18] A.J.C. Ladd, J. Fluid Mech. 271 (1994) 285.

[19] U. Frisch, D. d'Humières, B. Hasslacher, P. Lallemand, Y. Pomeau, J.-P. Rivet, Complex Systems 1 (1987) 649.

[20] C.P. Lowe, D. Frenkel, A.J. Masters, J. Chem. Phys. 103 (1995) 1582.

[21] D. Frenkel, B. Smit, Understanding Molecular Simulation. From Algorithms to Applications, Academic Press, Boston, 1996.

[22] W.G. Hoover, F.H. Ree, J. Chem. Phys. 49 (1968) 3609.

[23] A.J.C. Ladd, J. Chem. Phys. 93 (1990) 3484.

[24] H. Mori, Prog. Theoret. Phys. 34 (1965) 399.

[25] B.J. Berne, G.D. Harp, Adv. Chem. Phys. 17 (1970) 63.

[26] J.M. Deutch, I. Oppenheim, Faraday Discuss. Chem Soc. 83 (1987) 1. 